SSM Annual Scientific Meeting

Oral presentations

Wednesday 15 September

COVID Epi \& Control, $13.00-15.25$

${ }^{*}$ High scoring abstract

\section{OP01 EVALUATING THE IMPACTS OF TIERED RESTRICTIONS INTRODUCED IN ENGLAND, DURING OCTOBER AND DECEMBER 2020 ON COVID-19 CASES: A SYNTHETIC CONTROL STUDY*}

${ }^{1}$ Xingna Zhang*, 'Gwilym Owen, ${ }^{2}$ Mark Green, ${ }^{1}$ lain Buchan, ${ }^{1}$ Ben Barr. ${ }^{1}$ Public Health, Policy and Systems, University of Liverpool, Liverpool, UK; ${ }^{2}$ Department of Geography and Planning, University of Liverpool, Liverpool, UK

\subsection{6/jech-2021-SSMabstracts.1}

Background In 2020, a second wave of COVID-19 cases unevenly affected places in England leading to the introduction of a tiered system of controls with different geographical areas subject to different levels of restrictions. Whilst previous research has examined the impact of national lockdowns on transmission, there has been limited research examining the marginal effect of differences in localised restrictions or how these effects vary between socioeconomic contexts. We therefore examined how Tier 3 restrictions in England implemented between October-December 2020, which included additional restrictions on the hospitality sector and people meeting outdoors affected COVID-19 case rates, compared to Tier 2 restrictions, and how these effects varied by level of deprivation.

Methods We used data on weekly reported COVID-19 cases for 7201 neighbourhoods in England and adjusted these for changing case-detection rates to provide an estimate of weekly SARS-CoV-2 infections in each neighbourhood. We identified those areas that entered Tier 3 restrictions at two time points in October and December, and constructed a synthetic control group of similar places that had entered Tier 2 restrictions, using calibration weights to match them on a wide range of covariates that may influence transmission. We then compared the change in weekly infections between those entering Tier 3 to the synthetic control group to estimate the proportional reduction of cases resulting from Tier 3 restrictions compared to Tier 2 restrictions, over a 4-week period. We further used interaction analysis to estimate whether this effect differed based on the level of socioeconomic deprivation in each neighbourhood and whether effects were modified by the prevalence of a new more infectious variant of SARS-CoV-2 (B.1.1.7) in each area.

Results The introduction of Tier 3 restrictions in October and December was associated with a $14 \%$ (95\% CI $10 \%$ to $19 \%$ ) and $20 \%$ (95\% CI $13 \%$ to $29 \%$ ) reduction in infections respectively, compared to the rates expected if only Tier 2 restrictions had been in place in those areas. We found that effects were similar across levels of deprivation and limited evidence that Tier 3 restrictions had a greater effect in areas where the new more infectious variant was more prevalent.

Discussion Compared to Tier 2 restrictions, additional restrictions on hospitality and meeting outdoors introduced in Tier
3 areas in England had a moderate effect on transmission and these restrictions did not appear to increase inequalities.

\section{OP02 A COUNTERFACTUAL ANALYSIS OF THE EFFECTS OF LOCKDOWN TIMING ON CASES OF COVID-19 ACROSS EUROPE} ${ }^{1,2}$ Kellyn Arnold*, ${ }^{1,3,4}$ Peter Tennant, ${ }^{1,2,3}$ Alison Heppenstall, ${ }^{1,3,4}$ Mark Gilthorpe. 'Leeds
Institute for Data Analytics, University of Leeds, Leeds, UK; ${ }^{2}$ School of Geography, University
of Leeds, Leeds, UK; ${ }^{3}$ The Alan Turing Institute, London, UK; ${ }^{4}$ School of Medicine, University
of Leeds, Leeds, UK

\subsection{6/jech-2021-SSMabstracts.2}

Background Throughout the COVID-19 pandemic, many countries have relied on population-wide 'lockdown' measures to cut chains of transmission and prevent health services from being overwhelmed. Because of their substantial social and economic costs, these policies have typically only been implemented when it becomes clear that less stringent measures are insufficient to control virus spread. Nevertheless, the consequences of delaying lockdowns can be even more costly. In this study, we sought to quantify the extent to which delaying implementation of lockdown measures increases total case numbers and ultimately prolongs the length of lockdown required, by considering the first wave of cases across Europe.

Methods Data pertaining to COVID-19 cases and containment/ closure policies across Europe were obtained from online repositories maintained by Johns Hopkins and Oxford Universities, respectively. We identified key policy dates in each country, including the first date for which any restrictions were recommended or required, the date of lockdown, and the date lockdown was eased. We estimated the parameters governing the growth of COVID-19 during the first wave within each country, using linear splines fit to incident cumulative cases. To allow for standardised comparisons across countries, we considered three distinct periods of growth: initial uncontrolled growth, growth under recommended restrictions, and growth under lockdown. Using stochastic simulations, we then estimated: the natural (i.e. observed) growth of cases, and the counterfactual (i.e. hypothetical) growth of cases that would have been observed in each country had lockdown measures been implemented $1,3,5$, or 7 days earlier. Under each condition, we estimated the percentage change in total cases, and the percentage reduction in the required length of lockdown.

Results 39 European countries had both cases and policy data available, of which 33 entered lockdown and had estimable growth parameters. When lockdown was implemented 3 days earlier, total first wave cases were reduced by $16.93 \%$ (Q1Q3: 10.40-19.49) and the median required length of lockdown was reduced by $6.52 \%$ (Q1-Q3: 1.92, 10.53). When lockdown was implemented 7 days earlier, total first wave cases were reduced by $31.46 \%$ (Q1-Q3: 23.53-37.86) and the median required length of lockdown was reduced by $14.69 \%$ (Q1-Q3: 5.48, 24.40).

Conclusion Delaying implementation of national lockdown policies is likely to result in substantial case increases that ultimately prolong the length of lockdown required. Our results highlight the importance of acting swiftly to minimise the spread of COVID-19 when case numbers are increasing exponentially. 